\title{
Cyanobacteria as nanogold Factories: The chemical validation and perspective of the association between gold nanoparticles and cyanobacterial glycogen
}

\author{
Esam Bakir ( $\nabla$ ebakir@kfu.edu.sa ) \\ King Faisal University https://orcid.org/0000-0003-3566-3579 \\ Nermin ElSemary \\ King Faisal University
}

\section{Research}

Keywords: Cyanobacteria 1, Gold nanoparticles 2, Au-glycogen 3, HOMO and LUMO energy

Posted Date: July 27th, 2020

DOI: https://doi.org/10.21203/rs.3.rs-45902/v1

License: (c) (i) This work is licensed under a Creative Commons Attribution 4.0 International License. Read Full License 


\section{Abstract}

Background: Glycogen is the cyanobacterial reserve carbohydrate which is currently the focus of many studies. However, quantification of intercellular glycogen needs thorough investigation. The hypothesis is that glycogen can bind to nanogold. This binding can be used as an important tool for the quantification of intracellular glycogen.

Methods: Two strains of cyanobacteria were demonstrated to biosynthesise nanogold intracellularly and to bind to cellular glycogen. Then, spherical gold nanoparticles were chemically prepared and tested for binding to the glycogen molecule of cyanobacterial strains; Lyngbya majuscula and Cyanothece sp. via biochemical method. Experimental analyses were conducted to determine the morphological and optical properties of the Au-glycogen hydrocolloids, together with the analysis of the absorption spectra. The luminescence emission of AuNPs that resulted from recombination between electron in excited state HOMO and hole in ground state LUMO of gold nanoparticles according to Mie theory was recorded. The size diameter and shape of AuNPs were measured via scanning electron microscope and dynamic light scattering techniques. The stability of Au-glycogen was studied by the sequential addition of standard solutions of glycogen in the concentration range (10-100 $\left.\mu \mathrm{mol}^{-1}\right)$ into the prepared AuNPs colloidal solution by recording the SPR and luminescence intensity of AuNPs.

Results: The color of the cyanobacterial strains turned into purple color that indicated the formation gold nanoparticles inside the cell (intracellularly). To confirm binding between nanogold and glycogen, the absorption spectrum of AuNPs-glycogen showed plasmon band that was centered at $520-540 \mathrm{~nm}$, suggesting that gold nanoparticles were attached to the surface of the glycogen particles. The interaction of the gold nanoparticles with the biopolymer was further confirmed by photoluminescence spectroscopy analysis. The size diameter of the Au-glycogen in both Lyngbya majuscula and Cyanothece sp. were observed to be $41.7 \pm 0.2 \mathrm{~nm}$ and $80 \pm 30 \mathrm{~nm}$, respectively. FTIR analysis showed that the glycogen absorption peak was observed at 1,000 to $1,200 \mathrm{~cm}^{-1}$ and exhibited an increase corresponding to the increase in glycogen content in both cyanobacteria. In cyclic voltammetry scans, the $\mathrm{Au}^{3+} / \mathrm{Au}^{0}$ redox coupling was observed in case of Lyngbya majuscula indicating the formation of AuNPs-glycogen but in Cyanothece sp. the oxidation anodic peak of AuNPs disappeared which indicated that the AuNPs were highly stabilized in Lyngbya majuscula rather than in Cyanothece sp. This may be attributed to the presence of many thiazole peptides in Lyngbya majuscule. The luminescence of AuNPs showed more stability by the addition of gradual concentrations of glycogen and stronger emission of AuNPs as glycogen protected AuNPs agglomeration. The validation method applied to detect the concentration of glycogen was the use of the change in luminescence of AuNPs in correspondence to binding with glycogen. The detection limit (LOD) and quantitation limit (LOQ) were observed to be 0.89 and $2.95 \mu \mathrm{mol}$ $\mathrm{L}^{-1}$ respectively. Correlation convention $(\mathrm{R})$ was 0.995 . The good chemical stability of this colloidal system and the glycogen biomolecules are studied via density functional theory (DFT). The HOMO level of glycogen unit was closed near to LUMO level of $\mathrm{Au}^{3+}$ that supported the bioconversion of $\mathrm{Au}^{3+}$ into AuNPs via glucose units of glycogen. 
The detection limit (LOD) and quantitation limit (LOQ) were observed to be 0.89 and $2.95 \mu \mathrm{mol} \mathrm{L}^{-1}$ respectively, with $\mathrm{R}$ (correlation convention) equal to 0.995 . Computational calculations such as density functional theory (DFT) was used to confirm the Au-glycogen complex in bio-system. The HOMO level of glycogen unit was closed near to LUMO level of $\mathrm{Au}^{3+}$ that supported the bioconversion of $\mathrm{Au}^{3+}$ into AuNPs via glucose units of glycogen.

Conclusion: The associations formed between the gold nanoparticles and glycogen resulted in good chemical stability. The aggregation of the gold nanoparticles is related to the glycogen concentration and has a profound influence on the absorption properties of Au-glycogen systems. The interparticle distance between AuNPs and glycogen molecule induced the shift in the plasmon band.

\section{Background}

Cyanobacteria are autotrophic prokaryotes that possess chlorophyll a and perform oxygenic photosynthesis similar to plants and eukaryotic algae. In the calvin cycle, glycogen is stored in cyanobacteria Ball, [1, 2]. Chemically, glycogen is composed of glucose units which are attached via $a(1 \rightarrow 4)$ and $a(1 \rightarrow 6)$ glycosidic bonds. Glycogen has $70-80 \%$ of its structure as branched amylopectin and amylose. The chains in glycogen are randomly organized as reported by Manners [3]. Indeed, the chemical structure of glycogen was confirmed via the enzymatic analysis where glucose residues in the glycogen molecule are organized in a structure similar to a grape "Whelan-structure" [4]. Recently, the glycogen structure was revealed as a hyper-branched macromolecule with wide size distribution[5]. The branching and solubility of glycogen aid in the fast energy supply [6]. In cyanobacteria, the cycle of glycogen biosynthesis starts with the glucose-6-phosphate that is converted into ADP-glucose by phosphoglucomutase and ADP-glucose pyrophosphorylase. The glucose moiety in ADP-glucose is converted to the non-reducing end of the a-1,4-glucan backbone of glycogen by one or more glycogen synthases (GlgA). Subsequently, a branching enzyme introduces the a-1,6-linked glucosyl linkage, which is further extended to generate the glycogen particle. In the dark, glycogen is broken down by glycogen phosphorylase, glycogen debranching enzymes, a-glucanotransferase, and malto-dextrin phosphorylase into phosphorylated glucose and free glucose. These feed into catabolic pathways, including the oxidative pentose phosphate pathway, the Embden-Meyerhof-Parnas pathway (glycolysis), and the Entner-Doudoroff pathway[7]. Moreover, several genes affecting the accumulation of glycogen have been identified. A notable example is the discovery that the putative histidine kinase PmgA and the non-coding RNA PmgR1 form a regulatory cascade and control the accumulation of glycogen. Other regulatory elements are also known to affect the accumulation of glycogen, including the alternative sigma factor $\mathrm{E}$ and the transcriptional factor CyAbrB210[8]. The glucose unit of glycogen played important role in the reduction of gold salt to gold nanoparticles. In our previous work [9] we revealed, that nanogram-level glycogen could induce the aggregation of AuNPs to generate an enhanced plasmon resonance lightscattering signal at about $550 \mathrm{~nm}$. The novelty of research was concentrated on the possibility of using nanogold-glycogen binding to quantify cellular glycogen. Sensing this binding is possible through its ability of altering surface plasmon. The formation of AuNPs-glycogen is achieved through the addition of 
AuNPs that interact with glycogen of both Lyngbya majuscule (indicated as Lm) and Cyanothece sp. (indicated as Cy sp.) cyanobacteria. Therefore, we hypothesise that glycogen quantification can be based on the changes induced in the surface plasmon properties of nanogold particles as they bind to glycogen. Those changes in surface plasmon are in direct proportionality to the quantity of cellular glycogen. Similarly, we hypothesise that luminescence spectral changes can be also used as an indicator of glycogen quantity. Furthermore, an analytical validation model is to be developed to explore glycogennanogold interactions using regression of calibration curve between luminescence of AuNPs and the gradual concentrations of standard glycogen. The limit of detection (LOD), limit of quantification (LOQ) and sensitivity of analytical method are determined from calibration curve. Computational calculations of DFT analysis are to be used to explain the reaction mechanism of Au-glycogen system. Thus, the present study provides the multiple validations for the hypothesis that can be built on for future applications used for cellular glycogen quantification.

\section{Experimental Section}

\section{Reagents}

Hydrogen tetrachloroaurate hydrate $\left(\mathrm{HAuCl}_{4} \cdot 3 \mathrm{H}_{2} \mathrm{O}, 99.9 \%\right.$ purity), glycogen Oster (>75\%, Mwt. 666.58), saline $(0.9 \% \mathrm{NaCl})$, PBS, alanine $\left(0.2\right.$ mole $^{-1}$ in $\left.\mathrm{H} 2 \mathrm{O}\right)$, histidine $\left(0.2\right.$ mole $^{-1}$ in $\left.\mathrm{H}_{2} \mathrm{O}\right)$ and $\mathrm{D}$-glucose were received from Sigma-Aldrich (St. Louis, MS, USA). The organic solvents such as ethanol, methanol, acetonitrile (ACN) and dimethyl formamide (DMF) were purchased from Sigma-Aldrich (St. Louis, MS, USA with highly purity $(>99 \%)$.

\section{The isolation of cyanobacterial strains a) Lyngbya majuscula and b) Cyanothecesp.}

Isolation and establishment of mono-cyanobacterial cultures was performed as previously explained in [9], with the F/2 medium [10].

\section{Characterization methods}

The shape and size diameter of the AuNPs (citrate method) and Au-glycogen nanoparticles in the cyanobacteria bio-conjugate system were measured via scanning electron microscopy (SEM) using a Philips CM100 instrument and dynamic light scatter (DLS) instrument model Nano DS, SN 161. FTIR analysis of the glycogen and glycogen-gold bioconjugates were measured on Bruker Alpha FT-IR. The absorption and photoluminescence spectra of the samples were measured on a spectrofluorometer models USB4000 and USB4000FL, resp. (Ocean Optics Devices, USA). The Gamry 5000 potentiostat in a three-electrode system with a standard $\mathrm{Ag} / \mathrm{AgCl}$ reference, Pt-wire counter electrode, and gold working electrode (size diameter, $2 \mathrm{~mm}$ ) was used to explain the electrochemical properties of AuNPs and AuNPsglycogen with scan rate was $100 \mathrm{mV} \cdot \mathrm{S}^{-1}$. [11]. The $\mathrm{pH}$ was optimized at 7.40 .

\section{Chemical stability of the glycogen-stabilized Au nanoparticles}


The changes in the band position and width of AuNPs upon addition of glycogen was measured by absorption, photoluminescence and cyclic voltammetry spectra. A standard solutions of glycogen in concentration range (10-100 $\left.\mu \mathrm{mol} \mathrm{L}^{-1}\right)$ was added to fixed concentration of AuNPs $\left(0.277 \mathrm{mmol} \mathrm{L}^{-1}\right)$ in

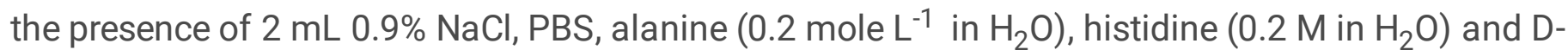

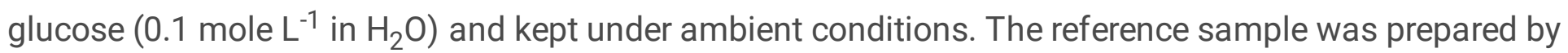
adding $2 \mathrm{~mL}$ of water into $2 \mathrm{~mL}$ of standard solution of AuNPs $0.277 \mathrm{mmol} \mathrm{L}^{-1}$ and glycogen $10 \mu \mathrm{mol} \mathrm{L}^{-1}$ solution. The experiments were replicated three times.

\section{Glycogen standard solutions and calibration}

The stock solution in the concentration $1 \mathrm{mmole}^{-1}$ was prepared by weighing specific amount of glycogen then dissolving it in distilled water. The calibrated solutions were in the concentration range of 10-100 umole $\mathrm{L}^{-1}$.

\section{Quantum chemistry of the Au-glycogen calculations.}

All geometries were optimized at the density functional (DFT) level via using B3LYP hybrid functional. For geometry optimization, density functional theory (DFT) can provide the most optimized structure of glycogen and gluconic acid. DFT was used to determine the molecular orbitals, energy band gaps and charge distribution maps [12]. The computations on this work were performed via using Gaussian09 codes. The basic set of DFT/B3LYP level of theory was 6G-31(d).

\section{Results And Discussion}

Glucose monomers were attached via glycosidic bonds to form a highly soluble branched polymer which is glycogen. Cyanobacteria, protozoa, fungi and animal cells all store glycogen as source of energy. Our results showed a bioconversion happened that changed gold salts into gold nanoparticles inside cyanobacteria cells. The binding and the correspondence between cellular glycogen and gold nanoparticles was tested by several analytical tools. The results will explain and demonstrate the interaction between $\mathrm{Au}^{3+}$ and glycogen and the spectral and optical changes that will follow upon which we based our hypothesis that nanogold can be used for quantifying cellular glycogen. The methods used for detecting those changes, thereby proving the hypothesis, are followed according to ICH analytical method validation parameters[13].

\section{Scanning electron microscope of Au-glycogen.}

Scanning electron (SEM) image showed the size diameter of AuNPs as well as presence of protein shell in Lyngbya majuscula cyanobacterial strain (Figure 1). The diameter of AuNPs was of an average size of $41.7 \pm 0.2 \mathrm{~nm}$ which was surrounded by protein shells of glycogen. The glycogen molecule contains glucose units of reducing property which are responsible for bio-reduction of $\mathrm{Au}^{3+}$ ions to AuNPs nanoparticles. The sulphur and thiol groups of thiazole peptides in L. majuscula chelated with AuNPs 
and aided in providing more stability of Au-glycogen[14]. The small size diameter of AuNPs was prepared by L. majuscula.

Dynamic light scattering (DLS) showed the large size diameter of purple AuNPs formed in Cyanothece sp. with size diameter of $80 \pm 30 \mathrm{~nm}$ [9]. Cyanothece sp. has large amount of thiol compounds which protect gold nanoparticles from agglomeration.

\section{The absorption and luminescence spectra of Au-glycogen.}

The plasmon band of AuNPs was recorded at 535 and $529 \mathrm{~nm}$ for Lyngbya majuscula and Cyanothece sp., respectively while the plasmon band of the prepared Au-glycogen was recorded at $524 \mathrm{~nm}$ (Figure 2a and $2 \mathrm{~b}$.). The position and shape of the plasmon band are dependent on the shape and size diameter of AuNPs where, for example, plasmon band beyond $600 \mathrm{~nm}$ are characteristic of non-spherical gold nanoparticles[15]. The narrow SPR band of AuNPs of Lyngbya majuscula confirmed the high stability of Au-glycogen bio-conjugated system and vice versa[16]. The photoluminescence (PL) emission of AuNPs was centered at 650-680 nm due to the recombination between the electron in e-LUMO and hole-HOMO of plasmonic effect of AuNPs which represented by Mie theory[17].The PL spectra of Au-glycogen in Cyanothecesp. was shifted to longer wavelength with compared the PL of prepared Au-glycogen due to the bigger size diameter of AuNPs[18].

\section{FTIR analysis Au-glycogen complex}

FTIR spectroscopy indicated that the glycogen biomacromolecules was attached to gold nanoparticles. The formation of Au-glycogen complex in Cyanothece sp. and L. majuscula cyanobacteria as bio-system was confirmed by FTIR analysis (Figure 3). The absorption bands of glycogen molecule were observed at 1,000 to $1,200 \mathrm{~cm}^{-1}[19]$. The absorption peaks intensity of Au-glycogen in L. majuscula was higher and narrower in bandwidth than those of Cyanothecesp. This is attributed to the higher contents of glycogen molecule in the former strain [20]. The glycogen biomolecule and AuNPs nanoparticles was linked through $\mathrm{OH}$ groups[16]. As glycogen quantity increased, the plasmon resonance peaks (SPR) increased in direct proportionality. This indicates that the inter- and intra-molecular $\mathrm{H}$-bonding in L. majuscula is formed as a result of complexation of glycogen molecule with the AuNPs. This is also supported by the observation that the absorption peak of $\mathrm{v}_{\mathrm{C}=0}$ stretching was shifted. This shift is suggested to be induced by inter-particle distance between AuNPs and glycogen molecule induced the shift in the plasmon band. This only reinforces the hypothesis of H-bonding in Au-glycogen complex [21]. The sulphur and thiol groups of thiazole peptides in L. majuscula are thought to be chelated to AuNPs and to provide more stability of Au-glycogen [14]. Hence, these FTIR data supplied an evidence on the formation of Auglycogen bio-conjugated complex which are also supported by the SEM images where the protein shell of glycogen was observed to be surrounded AuNPs as well.

\section{Cyclic-Voltammetry}


Cyclic voltammograms (CV) for Au-glycogen complex of both cyanobacteria was measured the rate of CVs scan $100 \mathrm{mV} / \mathrm{s}$ (Figure 4). The anodic and cathodic cycles of oxidation/ reduction potential peaks of Au-glycogen were recorded for $L$. majuscula around $0.8 \mathrm{~V}$ and $0.4 \mathrm{~V}$ (vs. $\mathrm{Ag} / \mathrm{AgCl}$ ), respectively. In Cyanothece sp. the anodic peak of Au-glycogen faded and the positive shifting of cathodic peak was shown around at $+0.60 \mathrm{~V}$ (vs. Ag/ $\mathrm{AgCl}$ )[22] due to the fast oxidation of AuNPs to Au-oxide ions with a lack of capping agent outside of cyanobacterial cells [23].

\section{The chemical stability of AuNPs-glycogen conjugated}

The stability of Au-glycogen complex was studied by the recording the luminescence intensity of the Auglycogen in excited state where only Au-glycogen system had luminescence to explicate the matrix interferences in bio system[16](Figure 5a.).

The analytical validation of calibration plotting (Figure $5 \mathrm{~b}$ ) was measured by adding standard solutions of glycogen in concentration range of $10-100 \mu \mathrm{mol} \mathrm{L}^{-1}$ and mixed with $0.277 \mathrm{mmol} \mathrm{L}^{-1}$ of AuNPs solution. The luminescence intensity of SPR* peak of AuNPs was increased by adding a more concentration of glycogen into AuNPs due to glycogen was stabilized the AuNPs excited state [16, 19]. The detection limit (LOD) and quantitation limit (LOQ) of the calibration curve of Au-glycogen were observed 0.89 and 2.95 $\mu \mathrm{mol} \mathrm{L} \mathrm{L}^{-1}$ respectively, with $\mathrm{R}$ (correlation convention) was 0.995 .

The analytical method has a good sensitivity for determination the glycogen concentration via the increasing of PL spectra of AuNPs (see Table1.).

Table 1 The sensitivity and regression parameters for the prepared Au-glycogen.

\begin{tabular}{|ll|}
\hline Analyte & Glycogen molecule concentration[a] \\
\hline Wavelength $(\mathrm{nm})$ & 633.00 \\
\hline Beers law limits & $1.00-100.0$ \\
\hline Regression line & $\mathrm{Y}=\mathrm{a}+\mathrm{bx}(\mathrm{a}=2.78, \mathrm{~b}=5795.00)$ \\
\hline Correlation coefficient $(\mathrm{R})$ & 0.995 \\
\hline LOD & 0.89 \\
\hline LOQ & 2.95 \\
\hline Sensitivity & $2.78 * 106$ \\
$(\varepsilon$, L mol-1 cm- 1$)$ & \\
\hline R.S.D $\%$ & 0.80 \\
\hline
\end{tabular}

[a] concentration $\left(\mu \mathrm{mol} \mathrm{L} \mathrm{L}^{-1}\right)$. 
The glycogen in cyanobacteria played important role for bioconversion of $\mathrm{Au}^{3+}$ into AuNPs in cell due to the reducing property. DFT calculations are used to calculate the HOMO, LUMO and energy gaps of $\mathrm{AuCl}_{4}$, glycogen and gluconic acid. The energy gap is energy difference between HOMO and LUMO orbitals according to Koopmans' theorem[24]. see eqn (1)).

\section{$\boldsymbol{E}_{\boldsymbol{g}}=\left(\boldsymbol{E}_{\text {Номо }}-\boldsymbol{E}_{\text {LUMO }}\right)$}

The energy gap was used to illustrate the molecular electrical transport properties between glycogen and $\mathrm{AuCl}_{4}$. The HOMO, LUMO and energy gap were interested to detect the possibility of electron transfer between glycogen molecule and gold ions were given in Table 2 where the energy HOMO level of glycogen molecule was closed near to LUMO level of $\mathrm{AuCl}_{4}$ which confirmed the electron transfer between glycogen molecule to $\mathrm{Au}^{3+}$ ions in ground state with formation gold nanoparticles [25].

Table 2. represented the HOMO, LUMO and energy gaps of AuCl4, glycogen molecule and gluconic acid.

\begin{tabular}{|lccc|}
\hline Compounds & $\begin{array}{l}\text { HOMO } \\
(\mathrm{eV})\end{array}$ & $\begin{array}{l}\text { LUMO } \\
(\mathrm{eV})\end{array}$ & $\begin{array}{l}\mathrm{Eg} \\
(\mathrm{eV})\end{array}$ \\
\hline $\mathrm{AuCl}_{4}$ & -8.7 & -6.5 & -2.2 \\
\hline Glycogen molecule & -5.3 & -4.9 & -0.4 \\
\hline Gluconic acid & -4.4 & -3.9 & -0.5 \\
\hline
\end{tabular}

The isodensity surfaces of $\mathrm{HOMO}$ and LUMO of glycogen molecule were shown the charge distribution in $\mathrm{CHO}, \mathrm{C}-\mathrm{O}$ groups as donor groups and $\mathrm{Au}$ in $\mathrm{AuCl}_{4}$ as accepter atom which was confirmed the conversion of $\mathrm{Au}^{3+}$ into AuNPs nanoparticles. (see Figure 3.6). The good electron injection properties of the glycogen molecule were observed the varied distributions of the HOMO and LUMO with various donor and acceptor levels.

\section{The proposed mechanism of the formation AuNPs-glycogen in cyanobacteria}

The mechanism of intra and extracellular reduction gold ions to AuNPs nanoparticles was facilitated by the interaction with glycogen, which most likely performs the role of electric wire that facilitates electron transfer [26]. The glycogen units have glucose units with strong reducing property which aids the bioreduction of gold ions into AuNPs in cyanobacteria [27]. The mechanism of the bioconversion of $\mathrm{Au}^{3+}$ into AuNPs was explained in the following steps. Firstly, the electrostatic interaction between $\mathrm{Au}^{3+}$ ions and glucose units in entities such as glycogen protein shell at the cell wall. Secondly, the reduction of the was taken place and AuNPs was aggregated with glucose unit was converted to gluconic acid. [28]. The microbial cell reduces metal ions by use of specific reducing enzymes like $\mathrm{NADH}$-dependent reductase or nitrate dependent reductase [29]. 
In Summary, it can be concluded that there is an association between gold nanoparticles and cellular glycogen concentration. This association has a profound influence on the absorption properties of Auglycogen systems as the inter-particle distance between AuNPs and glycogen molecule induced a shift in the plasmon band. The direct relationship between the concentration of glycogen and nanogold luminescence was analytically validated where the luminescence of AuNPs, denoted by detection limit (LOD) and quantitation limit (LOQ), had values of 0.89 and $2.95 \mu \mathrm{mol} \mathrm{L}^{-1}$, respectively. The correlation convention was quite high $(\mathrm{R}=0.995)$. Density functional theory (DFT) was used to explain the chemical stability of AuNPs-glycogen conjugate in cyanobacteria and both HOMO level of glycogen unit and LUMO level of $\mathrm{Au}^{3+}$ were close indicating excellent injection of electrons between them which overall supported the bioconversion of $\mathrm{Au}^{3+}$ into AuNPs via glucose units of glycogen.

\section{Conclusions}

All experimental data reinforced the hypothesis that there is stable association formed between the gold nanoparticles and glycogen. Hence, this can be used to quantify the amount of intercellular glycogen in cyanobacteria.

\section{Declarations}

\section{Ethical approval and consent to participate}

Not applicable (No human materials, data or participants were involved).

\section{Consent of publication}

All authors agreed to publication. No written permission is needed from a third party.

\section{Availability of data and materials}

All data generated or analysed during this study are included in this published article.

\section{Funding}

This research was funded by Deanship of Scientific Research, King Faisal University

Authors' contribution: Dr Bakir was responsible for the Chemical analyses, analysis and interpretation of data in addition to participation in the writing-up of manuscript. Professor El Semary was responsible for cyanobacterial isolation and culturing, participation in the writing up and obtaining the funding.

Acknowledgement: The authors express their deepest gratitude for the Deanship of Scientific Research , King Faisal University P.O box 400, Al-Ahsa, post code: 31982, Kingdom of Saudi Arabia grant number [17122009] for financial and moral support.

Conflict of interest: No conflict of interest exists 


\section{References}

1. Ball S, Guan H-P, James M, Myers A, Keeling P, Mouille G, Buléon A, Colonna P, Preiss J: From Glycogen to Amylopectin: A Model for the Biogenesis of the Plant Starch Granule.Cell 1996, 86:349352.

2. Cenci U, Nitschke F, Steup M, Minassian BA, Colleoni C, Ball SG: Transition from glycogen to starch metabolism in Archaeplastida. Trends in Plant Science 2014, 19:18-28.

3. Manners DJ: Recent developments in our understanding of glycogen structure. Carbohydrate Polymers 1991, 16:37-82.

4. Gunja-Smith Z, Marshall JJ, Mercier C, Smith EE, Whelan WJ: A revision of the Meyer-Bernfeld model of glycogen and amylopectin.FEBS Letters 1970, 12:101-104.

5. Fernandez C, Rojas CC, Nilsson L: Size, structure and scaling relationships in glycogen from various sources investigated with asymmetrical flow field-flow fractionation and 1H NMR.International Journal of Biological Macromolecules 2011, 49:458-465.

6. Meléndez R, Meléndez-Hevia E, Mas F, Mach J, Cascante M: Physical Constraints in the Synthesis of Glycogen That Influence Its Structural Homogeneity: A Two-Dimensional Approach.Biophysical Journal 1998, 75:106-114.

7. Wushensky JA, Youngster T, Mendonca CM, Aristilde L: Flux Connections Between Gluconate Pathway, Glycolysis, and Pentose-Phosphate Pathway During Carbohydrate Metabolism in Bacillus megaterium QM B1551.Frontiers in microbiology 2018, 9:2789.

8. De Porcellinis A, Frigaard N-U, Sakuragi Y: Determination of the glycogen content in cyanobacteria.JoVE (Journal of Visualized Experiments) 2017:e56068.

9. Younis NS, Bakir EM, Mohamed ME, El Semary NA: Cyanobacteria as Nanogold Factories II: Chemical Reactivity and anti-Myocardial Infraction Properties of Customized Gold Nanoparticles Biosynthesized by Cyanothece sp.Marine drugs 2019, 17:402.

10. Guillard RRL, Ryther JH: Studies of marine planktonic diatoms: I. Cyclotella nana Hustedt, and Detonula confervacea (Cleve) Gran.Canadian journal of microbiology 1962, 8:229-239.

11. Reddy MN, Srivastava V, Patil V: Effect of cadmium, lead and zinc on growth of some cyanobacteria.Journal of Ecobiology 2002, 14:161-167.

12. Gurdal Y, Iannuzzi M: DFT-based Theoretical Simulations for Photocatalytic Applications Using TiO2. In Titanium Dioxide. IntechOpen; 2017.

13. Agency EM: ICH Topic Q 2 (R1) validation of analytical procedures: text and methodology. EMEA London; 1995.

14. Amagata T: Natural Products Structural Diversity-II Secondary Metabolites: Sources, Structures and Chemical Biology.Comprehensive Natural Products // 2010, 2:581-621.

15. Amendola V, Pilot R, Frasconi M, Maragò OM, latì MA: Surface plasmon resonance in gold nanoparticles: a review.Journal of Physics: Condensed Matter 2017, 29:203002. 
16. Božanić DK, Luyt AS, Trandafilović LV, Djoković V: Glycogen and gold nanoparticle bioconjugates: controlled plasmon resonance via glycogen-induced nanoparticle aggregation. RSC advances 2013, 3:8705-8713.

17. Mie G: A contribution to the optics of turbid media, especially colloidal metallic suspensions. Ann Phys 1908, 25:377-445.

18. Huang Y, Fuksman L, Zheng J: Luminescence mechanisms of ultrasmall gold nanoparticles.Dalton Transactions 2018, 47:6267-6273.

19. Xiang M, Xu X, Li D, Liu F, Li N, Li K: Selective enhancement of resonance light-scattering of gold nanoparticles by glycogen. Talanta 2008, 76:1207-1211.

20. Welkie DG, Lee B-H, Sherman LA: Altering the structure of carbohydrate storage granules in the cyanobacterium Synechocystis sp. strain PCC 6803 through branching-enzyme truncations.Journal of bacteriology 2016, 198:701-710.

21. Talbott CM, Au-NP S: Spectroscopic Characterization of Nanoparticles for Potential Drug Discovery.

22. Wang Y, Laborda E, Crossley A, Compton RG: Surface oxidation of gold nanoparticles supported on a glassy carbon electrode in sulphuric acid medium: contrasts with the behaviour of 'macro'gold.Physical Chemistry Chemical Physics 2013, 15:3133-3136.

23. Welkie DG, Sherman DM, Chrisler WB, Orr G, Sherman LA: Analysis of carbohydrate storage granules in the diazotrophic cyanobacterium Cyanothece sp. PCC 7822.Photosynthesis research 2013, 118:25-36.

24. Koopmans $\mathrm{T}$ : The classification of wave functions and eigen-values to the single electrons of an atom.Physica 1934, 1:104-113.

25. Caruso F, Atalla V, Ren X, Rubio A, Scheffler M, Rinke P: First-principles description of charge transfer in donor-acceptor compounds from self-consistent many-body perturbation theory. Physical Review $B$ 2014, 90:085141.

26. Lovley DR: Extracellular electron transfer: wires, capacitors, iron lungs, and more.Geobiology 2008, 6:225-231.

27. Singh OV: Bio-nanoparticles: biosynthesis and sustainable biotechnological implications. John Wiley \& Sons; 2015.

28. Bansal V, Rautaray D, Ahmad A, Sastry M: Biosynthesis of zirconia nanoparticles using the fungus Fusarium oxysporum. Journal of Materials Chemistry 2004, 14:3303-3305.

29. Mandal D, Bolander ME, Mukhopadhyay D, Sarkar G, Mukherjee P: The use of microorganisms for the formation of metal nanoparticles and their application.Applied microbiology and biotechnology 2006, 69:485-492.

\section{Figures}




\section{protein shells}

$44.6 \mathrm{~nm}$

\section{$45.5 \mathrm{~nm}$}

$34.9 \mathrm{~nm}$

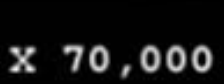

$15.0 \mathrm{kV}$ SEI

SBM

$100 \mathrm{~nm} \mathrm{KFU}-\mathrm{Sci}$

\section{Figure 1}

Scanning electron microscope image of Au-glycogen inside Lyngbya majuscula cyanobacteria.
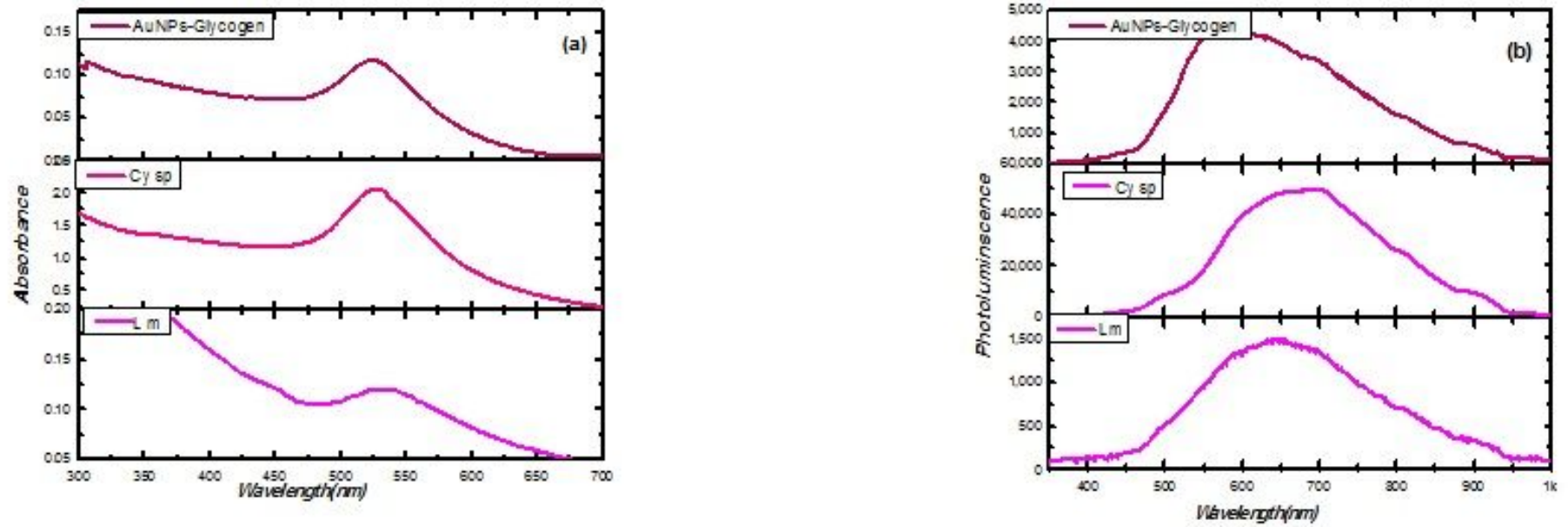
Figure 2

The absorption (a) and the photoluminescence spectra(b) of plasmon band of AuNPs when attached to glycogen of Lyngbya majuscula (Lm)and Cyanothece sp (Cy sp) and the prepared AuNPs-glycogen (top graph)

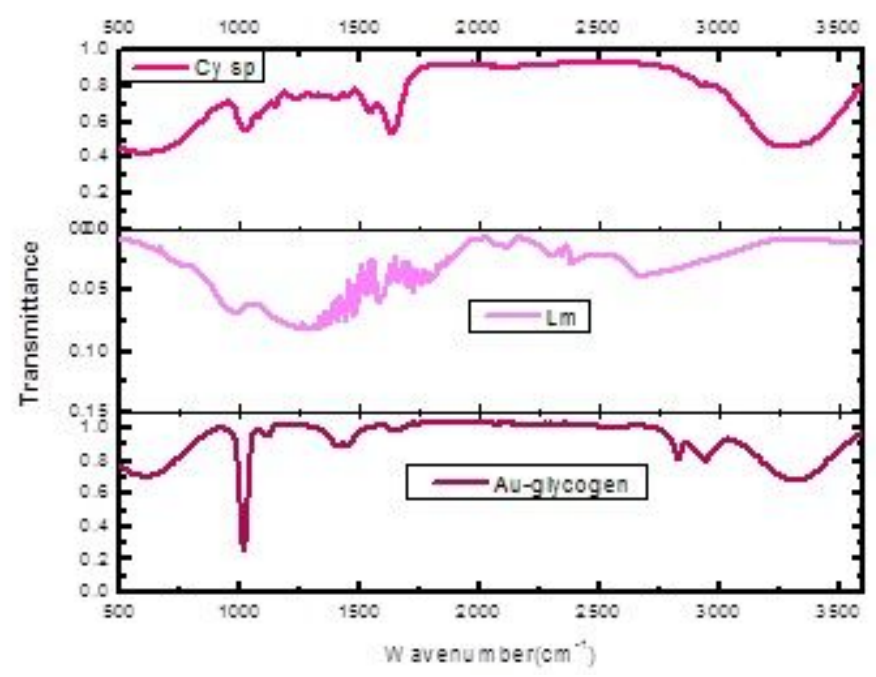

Figure 3

FTIR spectra of AuNPs-glycogen system with; Lyngbya majuscule (Lm) Cyanothece sp. (Cy sp) and the prepared Au-glycogen.

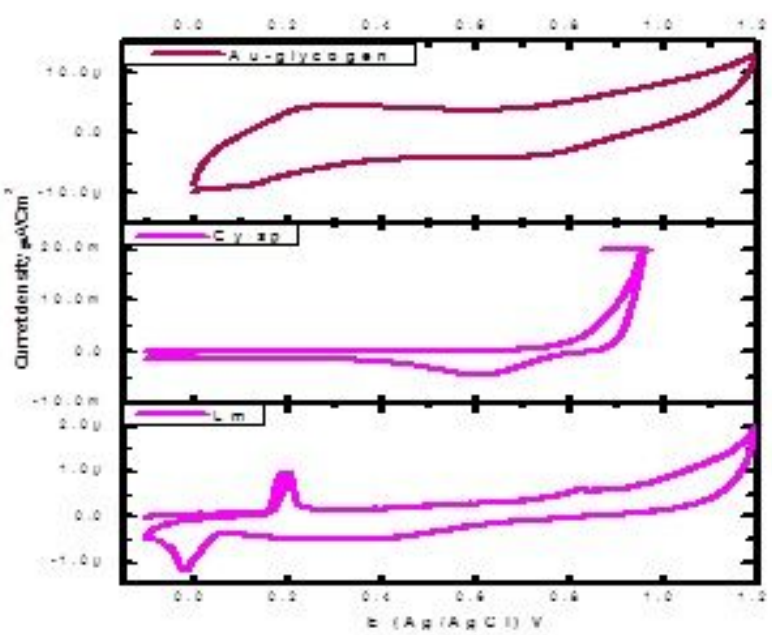

Figure 4

Cyclic-voltammgramms scans with scan rate $100 \mathrm{mv} / \mathrm{sec}$ of AuNPs-glycogen system with Lyngbya majuscula (Lm), Cyanothece sp (Cy sp) and the prepared Au-glycogen. 

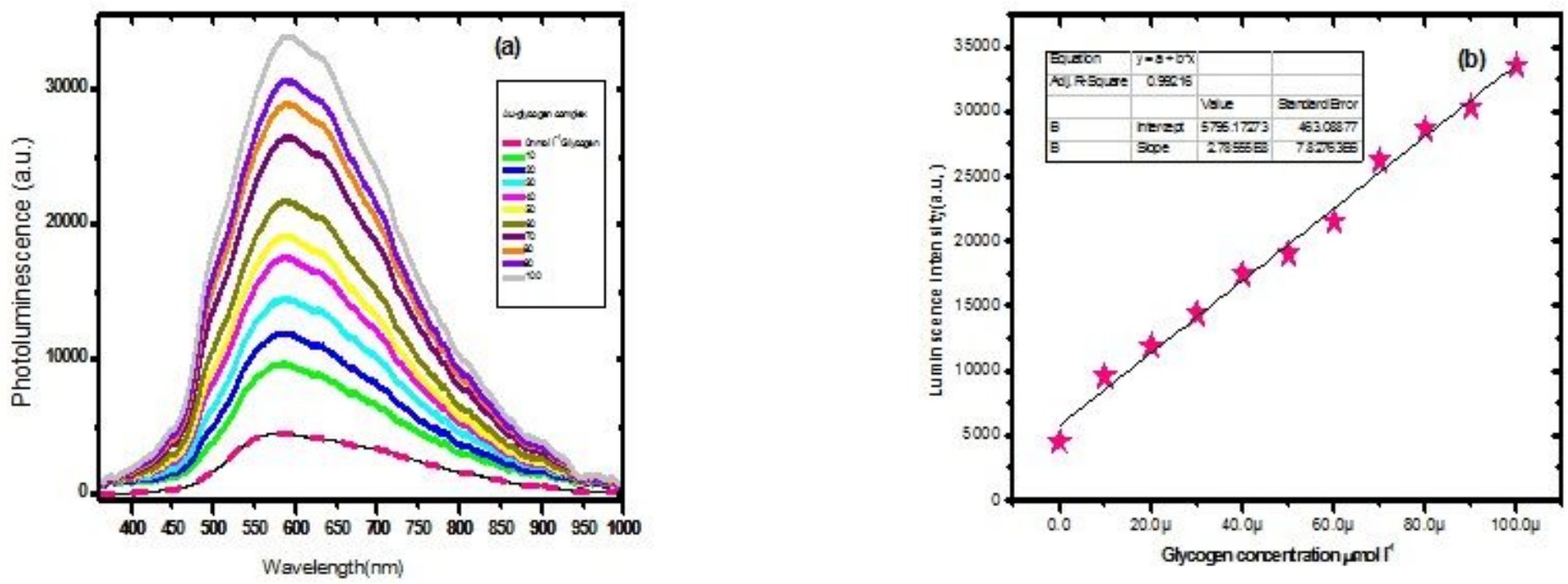

Figure 5

The photoluminescence spectra of AuNPs(a) and the calibration graph plotted(b) upon addition of 10$100 \mu \mathrm{mol}$ L-1 glycogen into colloidal AuNPs solution.

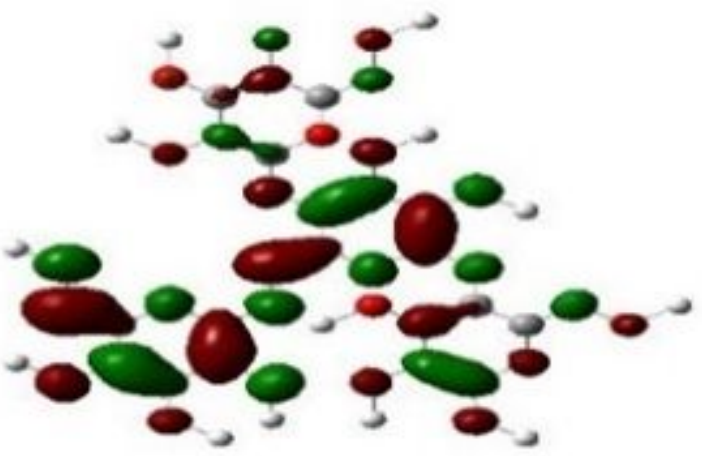

HOMO

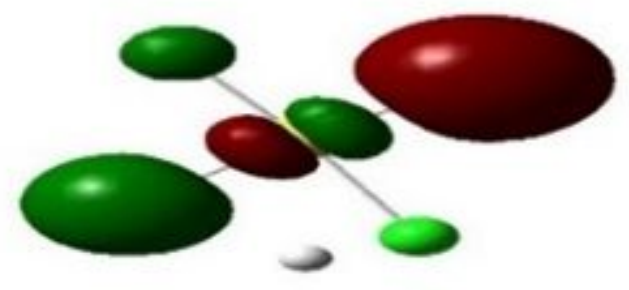

HOMO

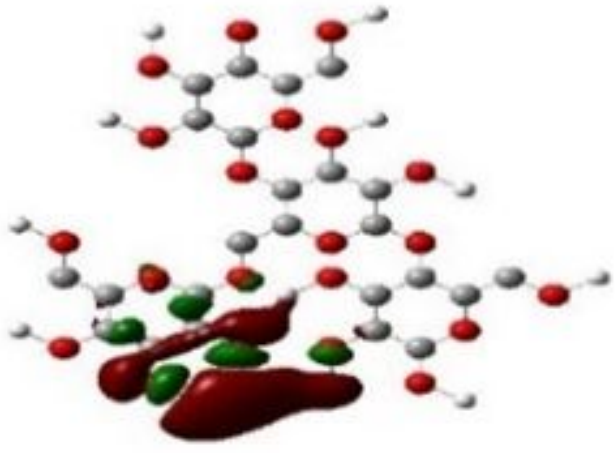

LUMO

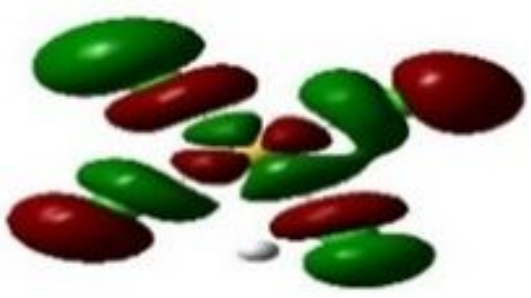

LUMO

Figure 6 
The isodensity surfaces of glycogen molecule and AuCl4 (HOMO and LUMO). 